\title{
p38 $y$ and p38 Mitogen Activated Protein Kinases (MAPKs), New Stars in the MAPK Galaxy
}

\author{
Alejandra Escós, Ana Risco, Dayanira Alsina-Beauchamp and Ana Cuenda * \\ Department of Immunology and Oncology, Centro Nacional de Biotecnología, Spanish National Research Council (CSIC), \\ Madrid, Spain
}

\section{OPEN ACCESS}

Edited by:

Matthias Gaestel,

MHH-Hannover Medical School,

Germany

Reviewed by:

Philippe P. Roux,

University of Montreal, Canada

Carl-Henrik Heldin,

Uppsala University, Sweden

Eulàlia De Nadal,

Pompeu Fabra University, Spain

*Correspondence:

Ana Cuenda

acuenda@cnb.csic.es

Specialty section:

This article was submitted to

Signaling,

a section of the journal

Frontiers in Cell and Developmental

Biology

Received: 29 January 2016 Accepted: 28 March 2016

Published: 14 April 2016

Citation:

Escós A, Risco A

Alsina-Beauchamp $D$ and Cuenda $A$ (2016) p38 $\gamma$ and p38s Mitogen Activated Protein Kinases (MAPKs),

New Stars in the MAPK Galaxy.

Front. Cell Dev. Biol. 4:31

doi: 10.3389/fcell.2016.00031
The protein kinases p38 $\gamma$ and p38 belong to the p38 mitogen-activated protein kinase (MAPK) family. p38MAPK signaling controls many cellular processes and is one of the most conserved mechanisms in eukaryotes for the cellular response to environmental stress and inflammation. Although p38 $\gamma$ and p38 8 are widely expressed, it is likely that they perform specific functions in different tissues. Their involvement in human pathologies such as inflammation-related diseases or cancer is starting to be uncovered. In this article we give a general overview and highlight recent advances made in defining the functions of p38 $\gamma$ and p38s, focusing in innate immunity and inflammation. We consider the potential of the pharmacological targeting of MAPK pathways to treat autoimmune and inflammatory diseases and cancer.

Keywords: p38 $\gamma, \mathrm{p} 38 \delta$, inflammation, innate response

\section{INTRODUCTION}

The activation of p38 Mitogen Activated Protein Kinases (p38MAPKs) is one of the main signal transduction mechanisms by which the cell adapts to changes in the environment. There are four p38MAPK isoforms in mammalian cells encoded by different genes: p38 $\alpha$ (MAPK14), p38 $\beta$ (MAPK 11), p38 (MAPK 12), and p38 (MAPK 13) (Cuenda and Rousseau, 2007). p38 $\alpha$ was the first p38MAPK family member identified, therefore the most studied and best-characterized isoform; most of the literature on $\mathrm{p} 38 \mathrm{MAPK}$ refers to $\mathrm{p} 38 \alpha$.

The four $\mathrm{p} 38 \mathrm{MAPK}$ isoforms are widely expressed, but their expression pattern varies in tissues. p38 $\alpha$ is ubiquitously expressed in all cell types and tissues, although expression levels are lower in the brain, liver, and pancreas than in other tissues. p38 $\beta$ is highly expressed in the brain, thymus, and spleen; its expression is lower in the adrenals, lung, kidney, liver, pancreas, and heart, and it is not expressed in skeletal muscle (Beardmore et al., 2005). p38 $\gamma$ is very abundant in skeletal muscle, although its expression in most other tissues is lower (Mertens et al., 1996; Beardmore et al., 2005). p38 levels are high in pancreas, intestine, adrenal gland, kidney, and heart (Goedert et al., 1997; Jiang et al., 1997; Beardmore et al., 2005).

p38MAPKs are strongly activated by a wide variety of environmental and cellular stresses or by inflammatory cytokines, but are poorly activated by serum or growth factors (Cuenda and Rousseau, 2007). All p38MAPKs are Serine/Threonine kinases that catalyze the reversible phosphorylation of proteins. They are activated by dual phosphorylation of the TGY activation motif mediated by the MAPK kinases (MAP2K) MKK3, and MKK6, and in the case of p38 $\alpha$ also by MKK4 (Remy et al., 2010). The activation of distinct p38MAPK isoforms is regulated by the selective and synchronized action of two kinases, MKK3 and MKK6. These two MAP2Ks 
are implicated in $\mathrm{p} 38 \alpha, \mathrm{p} 38 \gamma$, and $\mathrm{p} 38 \beta$ activation in response to general environmental stresses in mouse embryonic fibroblasts (Remy et al., 2010). However, MKK3 is the major kinase responsible for p38 $\delta$ activation (Remy et al., 2010). MKK3 and MKK6 are in turn activated upon phosphorylation of Serine/Threonine residues by a MAPK kinase kinase (MAP3K). Several MAP3Ks, including MAPK/ERK kinase kinases (MEKK), TAO1 and 2, ASK1 (apoptosis signal-regulating kinase-1), MLKs (mixed-lineage kinases), and TAK1 (TGF $\beta$-activated kinase 1) activate p38MAPK cascade; the specific MAP3K that is required appears to be stimulus and cell type specific (Cuenda and Rousseau, 2007) (Figure 1).

p38MAPK family can be further divided into two subsets, $\mathrm{p} 38 \alpha / \mathrm{p} 38 \beta$ and $\mathrm{p} 38 \gamma / \mathrm{p} 38 \delta$, based on sequence homology, substrate specificities, and sensitivity to chemical inhibitors. In this review, we provide an overview of p38 $\gamma$ and p $38 \delta$ (also called alternative p38MAPKs), which play important roles in the innate immune response, in inflammation and inflammation-related diseases such as cancer or arthritis.

\section{GENERAL FEATURES OF p38 $\gamma$ AND p38 $\delta$ SIGNALING PATHWAYS}

One of the hallmarks used for the division of p38MAPK into two subgroups is the protein sequence similarity. p38 $\gamma$ and $\mathrm{p} 38 \delta$ sequences are highly similar to each other ( $~ 70 \%$ identity), whereas $\mathrm{p} 38 \alpha$ and $\mathrm{p} 38 \beta$ display higher similarity (75\% identity). In contrast, $\mathrm{p} 38 \gamma$ and $\mathrm{p} 38 \delta$ are more divergent in sequence to p38 $\alpha$ ( $\sim 60 \%$ identical to p38 $\alpha$ ) (Risco and Cuenda, 2012). These two p38MAPK subgroups also differ on their sensitivity to kinase inhibitors. Pharmacological experiments demonstrated that only p38 $\alpha$ and p38 $\beta$ are inhibited by certain compounds, which are ATP competitors, such as SB203580 and other pyridinyl imidazoles, whereas p38 $\gamma$ and p38 $\delta$ are not affected by these drugs (Goedert et al., 1997; Kuma et al., 2005; Bain et al., 2007). However, the diaryl urea compound BIRB796, a molecule that functions allosterically and is a potent inhibitor of p38 $\alpha$ and p38 $\beta$, also inhibits p38 $\gamma$ and p38 8 at higher concentrations in cell-based assays. This compound has provided a good tool for identifying physiological substrates and roles of the alternative p38MAPK isoforms (Kuma et al., 2005; Cuenda and Rousseau, 2007; Risco and Cuenda, 2012). Nonetheless, due to the lack of specific p38 $\gamma$ and p38 inhibitors, the information about the physiological substrates and the biological function of these kinases is limited compared to the extensive knowledge of $\mathrm{p} 38 \alpha$ functions.

The use of kinase inhibitors and the genetic deletion of specific p38MAPK isoforms has showed that they have some overlapping substrates and functional redundancy; however, there are particular proteins that are better substrates for $\mathrm{p} 38 \alpha / \mathrm{p} 38 \beta$ than for $\mathrm{p} 38 \gamma / \mathrm{p} 38 \delta$ and the other way around (Kuma et al., 2005; Cuenda and Rousseau, 2007; Risco and Cuenda, 2012). Even more, protein kinases such as MAPK-activated protein kinase 2 (MK2) or MK3 are good substrates for p38 $\alpha$ and p38 $\beta$, but cannot be phosphorylated by other p38MAPK isoforms
(Cuenda et al., 1997; Goedert et al., 1997; Cuenda and Rousseau, 2007; Arthur and Ley, 2013) (Figure 1).

It has been reported that p38 kinase phosphorylates the neuronal microtubule-associated protein Tau (Feijoo et al., 2005), the eukaryotic elongation factor 2 kinase (eEF2K) (Knebel et al., 2001), the protein kinase D1 (PKD1) (Sumara et al., 2009), which controls insulin exocytosis in pancreatic beta cells and chemotaxis in neutrophils, and the signal adaptor p62, which controls mTORC1 activation, autophagy, and tumor growth (Linares et al., 2015).

Several physiological substrates for p38 $\gamma$ have been described taking advantage of a feature that makes p38 $\gamma$ unique among other MAPKs. p38 $\gamma$ possesses a short C-terminal sequence (-KETXL), which binds to PDZ domains. p38 $\gamma$ associates with PDZ-domain containing proteins, such as $\alpha 1$-syntrophin, SAP (synapse-associated protein) 90/PSD (post-synapse density) 95, hDlg (human disc large also known as SAP97) and the protein tyrosine phosphatase PTPH1 and under stress conditions it is able to phosphorylate them and modulate their activity (Hasegawa et al., 1999; Sabio et al., 2004, 2005; Hou et al., 2010). For example, changes in the osmolarity of the environment trigger p38 $\gamma$ activation in the cytoplasm, which phosphorylates hDlg. Phosphorylation of hDlg leads to its dissociation from the cytoskeletal guanylate kinase-associated protein (GKAP) and therefore from the cytoskeleton (Hasegawa et al., 1999; Sabio et al., 2004, 2005; Hou et al., 2010). In addition, the interaction of p38 $\gamma$ with the single PDZ domain of PTPH1 enables this phosphatase to dephosphorylate $\mathrm{p} 38 \gamma$, but not $\mathrm{p} 38 \alpha$, in vitro and in cells over-expressing both proteins (Hou et al., 2010; Chen et al., 2014). So far, the only physiological p38 $\gamma$ substrate that does not require PDZ domain binding interactions is the transcription factor $\mathrm{MyoD}$, whose phosphorylation by $\mathrm{p} 38 \gamma$ results in a decrease in its transcriptional activity (Gillespie et al., 2009).

p38MAPKs act normally by direct phosphorylation of substrates on Serine or Threonine residues followed by Proline, however, there are some examples showing that p38 $\alpha$ and also p38 $\gamma$ may also have kinase independent roles by associating to protein targets and modulating their function in the absence of phosphorylation (reviewed in Cuadrado and Nebreda, 2010; Risco and Cuenda, 2012). For example, it has been shown that $\mathrm{p} 38 \gamma$ regulates nuclear protein complexes independently of its kinase activity. Changes in the osmolarity cause the accumulation of $\mathrm{p} 38 \gamma$ in the nucleus where it interacts with nuclear $\mathrm{hDlg}$. In the nucleus, hDlg forms a complex with the proteins polypyrimidine tract-binding (PTB) protein-associated splicing factor (PSF) and p54nrb, and with various RNAs. $\mathrm{p} 38 \gamma$ regulates hDlg-PSF complex dissociation independently of hDlg phosphorylation by displacing PSF from hDlg, since both proteins, p38 $\gamma$ and PSF, bind to PDZ1 domain of hDlg. This has been shown comparing cells from knockin mice expressing an endogenous kinaseinactive $\mathrm{p} 38 \gamma$ mutant with cells from mice lacking p38 $\gamma$ (Sabio et al., 2005, 2010; Remy et al., 2010; Risco and Cuenda, 2012). The studies on p38 $\gamma$-hDlg-GKAP and p38 $\gamma$ hDlg-PSF protein complexes indicate that, through its ability to shuttle between cytoplasm and nucleus, p38 $\gamma$ might provide 

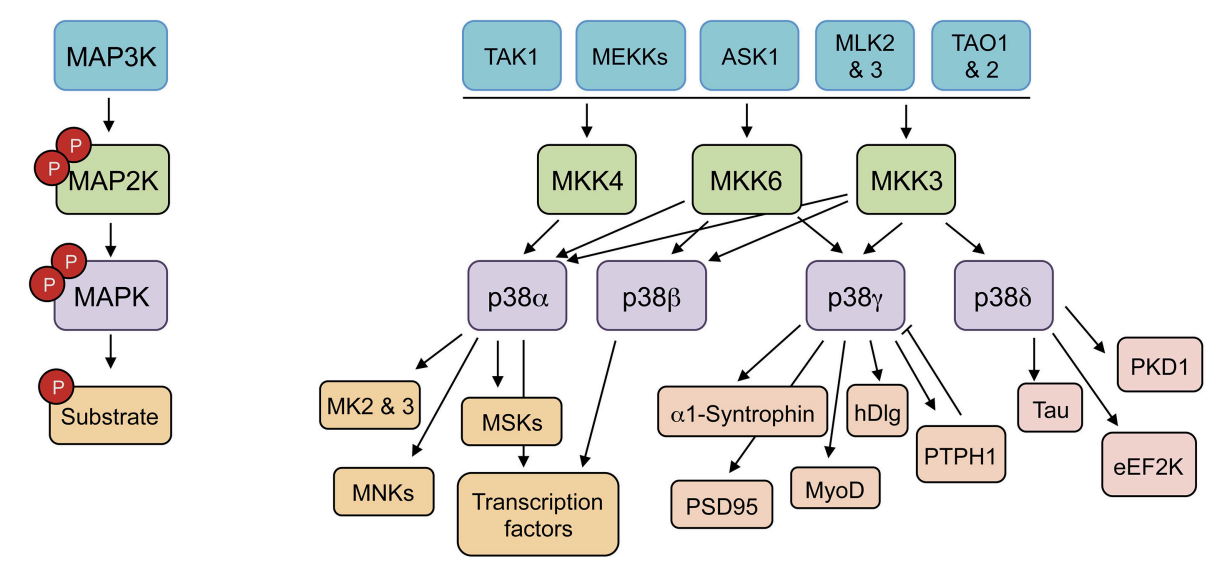

FIGURE 1 | p38MAPK pathways consist of several MAPK kinase kinases (MAP3K), three MAPK kinase (MAP2K), and four p38MAPK. These pathways can be activated by many stimuli, including growth factors, inflammatory cytokines, and a wide range of cellular stresses. The p38MAPKs phosphorylate different substrates, including protein kinases, cytosolic substrates, and transcription factors. ASK1 (apoptosis signal-regulating kinase-1), eEF2K (eukaryotic elongation factor 2 kinase), hDlg (human disc large), MEKK (MAPK/ERK kinase kinases), MK (MAPK-activated protein kinase), MLKs (mixed-lineage kinases), MNK (MAPK-interacting protein kinase), MSK (mitogen and stress-activated kinase), PKD1 (protein kinase D1), PSD95 (post-synapse density 95), PTPH1 (protein tyrosine phosphatase H1), TAK1 (TGF $\beta$-activated kinase 1), TAO (thousand-and-one amino acid).

a connection between two processes critical for adaptation to environmental changes: gene expression and cytoskeletal reorganization.

\section{SOME PHYSIOLOGICAL ROLES OF p38 $\gamma$ AND p38 $\delta$ MAPK PATHWAYS}

Studies using knock-out mice have provided important information concerning $\mathrm{p} 38 \gamma$ and $\mathrm{p} 38 \delta$ functions in vivo and in pathological conditions (Figure 2). p38 $\gamma$ and p $38 \delta$ deficient mice are viable and have not apparent phenotypes (Sabio et al., 2005, 2010; Remy et al., 2010; Risco and Cuenda, 2012). Nonetheless, there are reports showing the implication of $\mathrm{p} 38 \gamma$ and $\mathrm{p} 38 \delta$ in tissue regeneration, cancer, and metabolic diseases (Sabio et al., 2005, 2010; Remy et al., 2010; Risco and Cuenda, 2012). Thus, it has been described that $\mathrm{p} 38 \delta$ regulates insulin secretion and pancreatic $\beta$ cells death implying a central role in diabetes (Cuenda and Nebreda, 2009; Sumara et al., 2009). p38 is also crucial in neutrophil chemotaxis pathway, contributing to acute respiratory distress syndrome (ARDS) (Ittner et al., 2012), and in mediating IL-13-driven mucus overproduction in human airway epithelial cells in chronic inflammatory lung diseases (Alevy et al., 2012).

Since p38 $\gamma$ expression is very high in skeletal muscle and its expression is induced during muscle differentiation (Cuenda and Cohen, 1999; Tortorella et al., 2003; Perdiguero et al., 2007), it is not surprising that it plays a fundamental role in this process. Thus, p38 $\gamma$ knockdown impairs cardiomyocyte formation (Ramachandra et al., 2016) and p38 $\gamma$ and $\mathrm{p} 38 \delta$ promote cardiac hypertrophy by modulating the mTOR pathway (González-Terán et al., 2016). Moreover, studies in p38 $\gamma$ deficient mice reported that $\mathrm{p} 38 \gamma$ plays a pivotal role in blocking the premature differentiation of skeletal muscle stem cells, the satellite cells that participate in adult muscle regeneration (Gillespie et al., 2009). Also, p38 $\gamma$ is required for the upregulation of PGC- $1 \alpha$ [peroxisome proliferator-activated receptor- $\gamma(\operatorname{PPAR} \gamma)$ coactivator- $1 \alpha]$ in mitochondrial biogenesis and angiogenesis in response to endurance exercise in mice, which is critical for skeletal muscle adaptation (Pogozelski et al., 2009).

In addition, $\mathrm{p} 38 \gamma$ and $\mathrm{p} 38 \delta$ are involved in the modulation of some processes implicated in cellular malignant transformation, such as proliferation, cell cycle progression, apoptosis, or cell migration. Using mouse embryonic fibroblasts derived from mice lacking $\mathrm{p} 38 \gamma$ or $\mathrm{p} 38 \delta$, it has been shown that deletion of either p38 $\gamma$ or p38 increases cell migration and metalloproteinase2 secretion, whereas only p $38 \delta$ deficiency impairs cell contact inhibition. Also, lack of p38 $\gamma$ in K-Ras-transformed fibroblasts leads to increased cell proliferation as well as tumorigenesis both in vitro and in vivo (Cerezo-Guisado et al., 2011). These pieces of evidence indicates that $\mathrm{p} 38 \gamma$ and $\mathrm{p} 38 \delta$ have a role in tumor suppression; however, there are other studies reporting a pro-oncogenic function for these kinases, for example in the development of breast and skin cancer (reviewed in Risco and Cuenda, 2012).

\section{p38 $\gamma$ AND p388 IN THE INNATE RESPONSE AND IN INFLAMMATION}

The use of genetically modified mice lacking one or more p38 isoform has provided strong evidence of the $\mathrm{p} 38 \gamma$ and $\mathrm{p} 38 \delta$ importance in the innate immune response and in inflammation (Figure 2). The innate immune system is the front line of defense against invading pathogens, and uses evolutionarily conserved high-affinity receptors (pattern recognition receptors, PRRs) that recognize specific constituents of bacteria or virus, endogenous cytokines and host cell-derived components (Kawai and Akira, 2007). The activation of PRRs in the innate immune cells leads to 


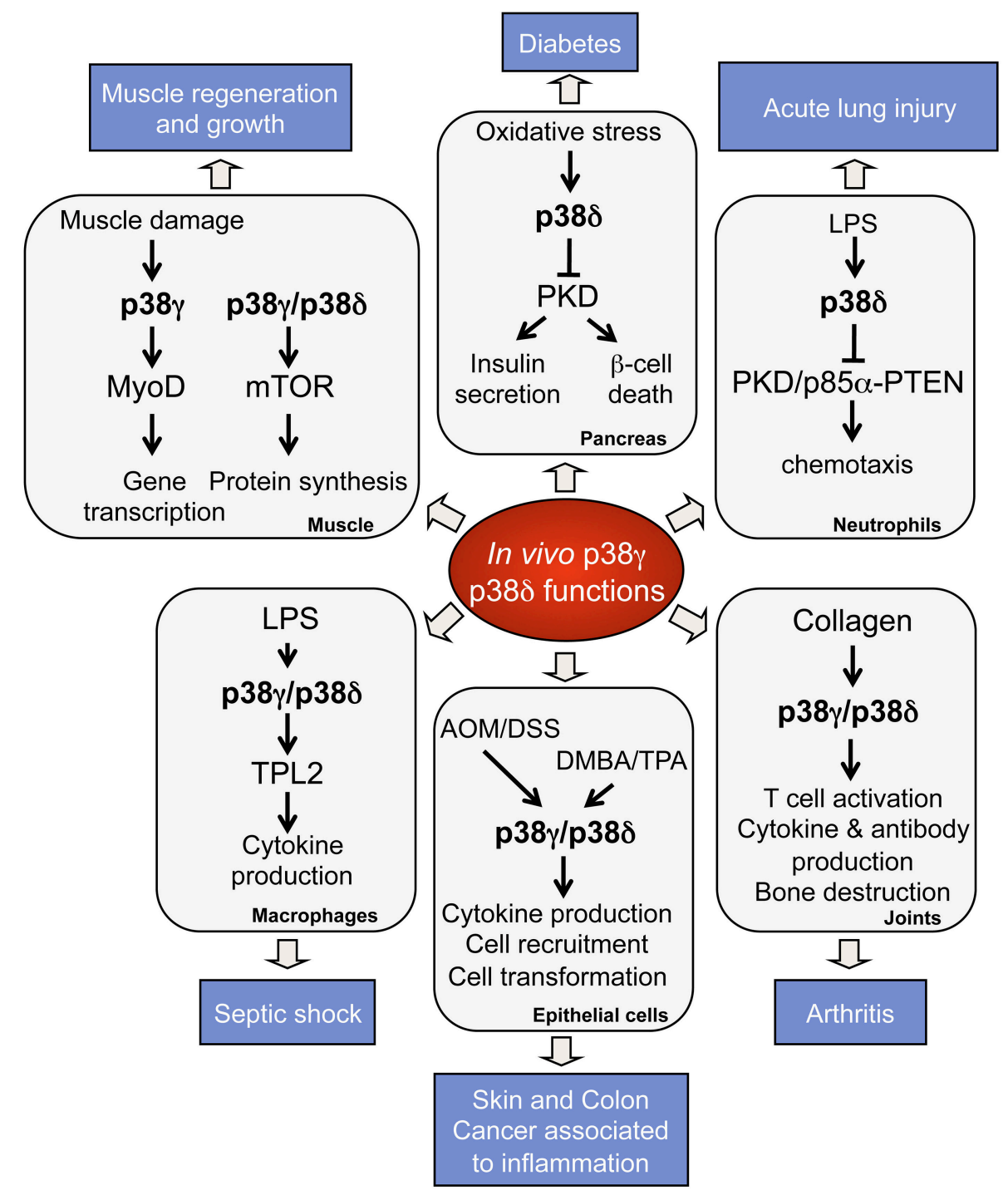

FIGURE 2 | Physiological roles and pathological implications of p38 $\gamma$ and p388. p38 $\gamma$ and p38 are key players in the regulation of many biological functions, which contribute to physiological processes. Deregulation of $\mathrm{p} 38 \gamma$ and $\mathrm{p} 388$ leads to the development of several pathological conditions.

secretion of inflammatory cytokines and other mediators, which induce an inflammatory response. This inflammatory response promotes the recruitment of additional immune cells, the elimination of infectious agents, and the induction of tissue repair (Kawai and Akira, 2007). The production of cytokines during the innate response is also important for the subsequent induction of the adaptive immune response (Iwasaki and Medzhitov, 2010). The stimulation of all PRRs by pathogen-associated molecules activates both MAPKs and NFKB pathways, which are crucial to generate immune responses (Cuenda and Rousseau, 2007; Gaestel et al., 2009; Arthur and Ley, 2013).

The important role of the MAPK p $38 \alpha$ in the innate immune response and in inflammation has been uncovered mainly by studies using a range of $\mathrm{p} 38 \alpha$ inhibitors or the constitutive deletion of its physiological substrates, or activators (Cuenda and Rousseau, 2007; Gaestel et al., 2009; Arthur and Ley, 2013). Much less is known about the importance of p38 $\gamma$ and p38 in these processes. However, recent studies show that in macrophages and dendritic cells (DC), which are key mediators of the inflammatory response, the deletion of both $\mathrm{p} 38 \gamma$ and p38 impaired the innate immune response to lipopolysaccharide (LPS), a Toll-like receptor 4 (TLR4) ligand (Risco et al., 2012). In these cells $\mathrm{p} 38 \gamma$ and $\mathrm{p} 38 \delta$ are necessary to maintain steadystate levels of TPL-2, the MKK kinase that mediates ERK1/2 activation in response to TLR stimulation (Gantke et al., 2011). ERK1/2 are other MAPK family members that play a central role in cytokine production. p38 $\gamma$ and p38 deficient macrophages ( $\mathrm{p} 38 \gamma / \delta^{-/-}$) showed substantially lower levels of TPL2 protein, and therefore lower MKK1-ERK1/2 activation and inflammatory cytokine production (Risco et al., 2012). Exogenous expression 
of TPL-2 in p38 $\gamma / \delta^{-/-}$macrophages not only increased ERK1/2 activation, but also rescued TPL-2-dependent TNF $\alpha$ production in response to LPS (Risco et al., 2012).

p38 $\gamma$ and p38 signaling has complex pro- and antiinflammatory effects on cytokine production in innate immune responses. Production of TNF $\alpha$, IL-1 $\beta$, and IL-10 is severely reduced in LPS-stimulated macrophages from $\mathrm{p} 38 \gamma / \delta$-deficient mice, whereas IL-12 and IFN $\beta$ production increases (Risco et al., 2012). $\mathrm{p} 38 \gamma$ and $\mathrm{p} 38 \delta$ regulate IL-1 $\beta$ and IL-10 production at the transcriptional level, whereas regulation of TNF $\alpha$ is at the secretion level in bone marrow derived macrophages stimulated with LPS (Risco et al., 2012). In LPS-stimulated liver macrophages, p38 $\gamma$ and p38 are required for the translation of Tnf mRNA through inhibitory phosphorylation of eEF2K that leads to activation of eEF2 (González-Teran et al., 2013). Furthermore, in TPA-stimulated keratinocytes, p38 $\gamma$ and p38 are required for IL-6, IL-1 $\beta$, and CXCL1 transcription (Zur et al., 2015). The exact mechanisms by which $\mathrm{p} 38 \gamma$ and $\mathrm{p} 38 \delta$ regulate the production of cytokines and chemokines in different cells are still largely unknown and further studies are needed to determine them.

Using p38 deficient mice it has been shown that this p38MAPK isoform is important in neutrophils migration and in their recruitment into inflammatory sites in lung (Ittner et al., 2012). The degree of inflammation and the associated organ damage is a consequence of complex proand anti-inflammatory responses, which involve the regulation of neutrophil recruitment and migration in a cell-autonomous manner. p388-deficient neutrophils show a defect in chemotaxis, which is caused by increased activity of the p $38 \delta$ substrate, the kinase PKD1 (Sumara et al., 2009). PKD1 phosphorylates p $85 \alpha$ to enhance its interaction with PTEN, leading to increased PTEN activity and lower cell migration (Ittner et al., 2012). Appropriate signaling in neutrophils is essential to resolve inflammation without causing inappropriate organ damage.

Overall, these data strongly suggest that $\mathrm{p} 38 \gamma$ and $\mathrm{p} 38 \delta$ have a key role in the mechanisms leading to inflammation.

\section{p38y AND p388 IN INFLAMMATORY DISEASES}

The role of $\mathrm{p} 38 \gamma$ and $\mathrm{p} 38 \delta$ in inflammation in vivo is further supported by experiments in other mouse animal models. The reaction to bacterial LPS is a well-characterized innate immune response that leads to endotoxic or septic shock, due primarily to TNF $\alpha$ overproduction. Thus, $\mathrm{p} 38 \gamma / \delta$-deficient mice are less sensitive to endotoxic shock than wild type mice following LPS challenge and this is associated with a decrease in serum levels of inflammatory cytokines such as TNF $\alpha$, IL- $1 \beta$, or IL10 (Risco et al., 2012). The acute liver failure caused by LPS is also suppressed in mice that lack p38 $\gamma$ and p $38 \delta$ in myeloid cells (González-Teran et al., 2013). In addition, p38 deletion results in decreased alveolar neutrophil accumulation, reduces acute lung inflammation, and protect from acute lung injury (ALI) induced by LPS (Ittner et al., 2012). Also, there are evidence that $\mathrm{p} 38 \delta$ mediates mucus production in chronic inflammatory lung disease, since either the knockdown or inhibition of $\mathrm{p} 38 \delta$, but not of $\mathrm{p} 38 \alpha$, can block inflammatory IL13-induced mucus production in human airway epithelial cells (Alevy et al., 2012).

The role of $\mathrm{p} 38 \gamma$ and $\mathrm{p} 38 \delta$ isoforms in other inflammatory diseases such as arthritis has recently been shown in a collageninduced arthritis (CIA) mouse model. Combined p38 $\gamma$ and $338 \delta$ deficiency markedly reduced arthritis severity by suppressing clinical disease and bone destruction, compared with that in wild type mice (Criado et al., 2014). p38 $\gamma / \delta$ deficient mice have lower mRNA expression of IL-17 and IFN $\gamma$ in joints, and lower levels of pathogenic anti-collagen antibodies, IL-1 $\beta$, and TNF- $\alpha$ in the serum than wild type mice (Criado et al., 2014). p38 $\gamma$ and p38 also seem to control $\mathrm{T}$ cell activation, for example lymph node $\mathrm{T}$ cells from $\mathrm{p} 38 \gamma / \delta$-deficient mice show reduced proliferation and interferon (IFN) $\gamma$ and IL-17 production (Criado et al., 2014). Moreover, p38 $\gamma / \delta$ deficient mice showed a lower Th17 cell frequency and a greater Treg/Th17 ratio, both of which are linked to successful therapy in rheumatoid arthritis. The crucial role of $\mathrm{p} 38 \gamma / \delta$ in synovial inflammation, bone erosion, as well as cytokine production suggests that they could serve as targets of therapy in rheumatoid arthritis as an alternative to traditional p38 $\alpha$ inhibitors, which have proven minimally effective in human disease (Gaestel et al., 2009; Arthur and Ley, 2013).

During the last few years the role of $\mathrm{p} 38 \gamma$ and $\mathrm{p} 388$ in cancer associated with chronic inflammation has been studied. Chronic inflammation is linked with an increase in malignant disease. Almost 20\% of human cancers are related to chronic inflammation caused by infections, exposure to irritants or autoimmune diseases (Hanahan and Weinberg, 2011; Crusz and Balkwill, 2015). Colitis-associated cancer (CAC) is a colon cancer subtype associated with inflammatory bowel disease, such as that occurring in ulcerative colitis or Crohn's disease. Using the azoxymethane (AOM)/dextran sodium sulfate (DSS) mouse model of CAC it has been shown that $\mathrm{p} 38 \gamma$ and $\mathrm{p} 38 \delta$ have a prooncogenic role by regulating inflammatory signaling to promote colon tumorigenesis, thus linking inflammation and cancer in CAC (Del Reino et al., 2014). Mice deficient in p38 $\gamma$ and p38 $\delta$ display a decrease in cytokines and chemokines production and in inflammatory cell infiltration in the colon of treated animal and produce fewer colon tumors than control mice (Del Reino et al., 2014). p38 $\gamma$ and p 388 in hematopoietic cells are important for CAC development. Lethally irradiated wild type mice reconstituted with bone marrow from $\mathrm{p} 38 \gamma / \delta$-null mice exhibited less tumor formation, cytokine production, and immune cell infiltration, whereas $\mathrm{p} 38 \gamma / \delta$-deficient mice reconstituted with wild type bone marrow showed more tumor formation, cytokine production, and immune cell infiltration than controls (Del Reino et al., 2014). The pro-oncogenic role of p38 $\gamma$ and p $38 \delta$ was also confirmed in the two-step 7,12-dimethylbenz(a)anthracene (DMBA)/12-O-tetradecanoylphorbol-13-acetate (TPA) chemical skin carcinogenesis model (Schindler et al., 2009; Zur et al., 2015). p38 $/ / \delta$-deficient mice showed diminished cytokine production and are resistant to tumorigenesis (Zur et al., 2015). Overall, all of these results suggest potential therapeutic prospects by targeting p $38 \gamma$ and $\mathrm{p} 38 \delta$ for treatment of cancer. Future studies examining the effects of cell type-selective p38 $\gamma$ and p38 targeting at different stages of carcinogenesis will elucidate the functional roles of these two alternative p38MAPKs in 
the tumorigenesis process, and will guide future therapeutic strategies.

\section{CONCLUSION AND PERSPECTIVE}

The role of $\mathrm{p} 38 \gamma$ and $\mathrm{p} 38 \delta$ has been some times ignored since most of the studies to date have focused on p38 $\alpha$, which is the most abundant p38MAPK isoform. Nonetheless, in the last years significant progress in understanding the functions of $\mathrm{p} 38 \gamma$ and $\mathrm{p} 38 \delta$ in vivo has been achieved. It is now clear that $\mathrm{p} 38 \gamma$ and p38 8 are crucial in innate response, inflammation, and inflammatory diseases. Therefore, they deserve to be studied in greater depth as they represent pharmacological target for the development of drugs that might be useful for the treatment of inflammatory pathologies. In fact, development of more specific p38 inhibitors has been shown to reduce mucus production in human airway epithelial cells (Alevy et al., 2012). However, the molecular mechanisms of how $\mathrm{p} 38 \gamma$ and $\mathrm{p} 38 \delta$ regulate innate response, including the significance of the regulation of other signaling pathways components, cytokine production and the recruitment of immune cells, remain to be fully established. A better mechanistic understanding of $\mathrm{p} 38 \gamma / \mathrm{p} 38 \delta$-regulated innate

\section{REFERENCES}

Alevy, Y. G., Patel, A. C., Romero, A. G., Patel, D. A., Tucker, J., Roswit, W. T., et al. (2012). IL-13-induced airway mucus production is attenuated by MAPK13 inhibition. J. Clin. Invest. 122, 4555-4568. doi: 10.1172/JCI64896

Arthur, J. S., and Ley, S. C. (2013). Mitogen-activated protein kinases in innate immunity. Nat. Rev. Immunol. 13, 679-692. doi: 10.1038/nri3495

Bain, J., Plater, L., Elliott, M., Shpiro, N., Hastie, C. J., McLauchlan, H., et al. (2007). The selectivity of protein kinase inhibitors: a further update. Biochem. J. 408, 297-315. doi: 10.1042/BJ20070797

Beardmore, V. A., Hinton, H. J., Eftychi, C., Apostolaki, M., Armaka, M., Darragh, J., et al. (2005). Generation and characterization of p38beta (MAPK11) genetargeted mice. Mol. Cell. Biol. 25, 10454-10464. doi: 10.1128/MCB.25.23.1045410464.2005

Cerezo-Guisado, M. I., del Reino, P., Remy, G., Kuma, Y., Arthur, J. S., Gallego-Ortega, D., et al. (2011). Evidence of p38gamma and p38delta involvement in cell transformation processes. Carcinogenesis 32, 1093-1099. doi: 10.1093/carcin/bgr079

Chen, K. E., Lin, S. Y., Wu, M. J., Ho, M. R., Santhanam, A., Chou, C. C., et al. (2014). Reciprocal allosteric regulation of p38gamma and PTPN3 involves a PDZ domain-modulated complex formation. Sci. Signal. 7:ra98. doi: 10.1126/scisignal.2005722

Criado, G., Risco, A., Alsina-Beauchamp, D., Pérez-Lorenzo, M. J., Escós, A., and Cuenda, A. (2014). Alternative p38 MAPKs are essential for collagen-induced arthritis. Arthritis Rheumatol. 66, 1208-1217. doi: 10.1002/art.38327

Crusz, S. M., and Balkwill, F. R. (2015). Inflammation and cancer: advances and new agents. Nat. Rev. Clin. Oncol. 12, 584-596. doi: 10.1038/nrclinonc.2015.105

Cuadrado, A., and Nebreda, A. R. (2010). Mechanisms and functions of p38 MAPK signalling. Biochem. J. 429, 403-417. doi: 10.1042/BJ20100323

Cuenda, A., and Cohen, P. (1999). Stress-activated protein kinase-2/p38 and a rapamycin-sensitive pathway are required for $\mathrm{C} 2 \mathrm{C} 12$ myogenesis. J. Biol. Chem. 274, 4341-4346. doi: 10.1074/jbc.274.7.4341

Cuenda, A., Cohen, P., Buée-Scherrer, V., and Goedert, M. (1997). Activation of stress-activated protein kinase-3 (SAPK3) by cytokines and cellular stresses is mediated via SAPKK3 (MKK6); comparison of the specificities of SAPK3 and SAPK2 (RK/p38). EMBO J. 16, 295-305. doi: 10.1093/emboj/16.2.295

Cuenda, A., and Nebreda, A. R. (2009). p38delta and PKD1: kinase switches for insulin secretion. Cell 136, 209-210. doi: 10.1016/j.cell.2009.01.005 response will permit the design of $\mathrm{p} 38 \gamma$ and $\mathrm{p} 38 \delta$-based therapies, alternative to traditional p38 $\alpha$ inhibitors, which have proven minimally effective in human inflammatory diseases (Gaestel et al., 2009; Arthur and Ley, 2013). The prospective that basic research on p38 $\gamma$ and $\mathrm{p} 38 \delta$ could be translated to the treatment of human disease provides an exciting goal for future studies in the field.

\section{AUTHOR CONTRIBUTIONS}

AC wrote the manuscript. AE, AR, DA made substantial contributions to conception and design, and acquisition of information. All authors contributor to the revision of the manuscript and approved the final version.

\section{ACKNOWLEDGMENTS}

The work in the author's laboratory is supported by grants from the Ministerio de Economia y Competitividad (MINECO) (SAF2013-45331-R) and La Marató TV3 Foundation (82031) to AC. AE and DA receive MINECO FPI fellowships and AR Juan de la Cierva award from MINECO.

Cuenda, A., and Rousseau, S. (2007). p38 MAP-kinases pathway regulation, function and role in human diseases. Biochim. Biophys. Acta 1773, 1358-1375. doi: 10.1016/j.bbamcr.2007.03.010

Del Reino, P., Alsina-Beauchamp, D., Escós, A., Cerezo-Guisado, M. I., Risco, A., Aparicio, N., et al. (2014). Pro-oncogenic role of alternative p38 mitogenactivated protein kinases p38gamma and p38delta, linking inflammation and cancer in colitis-associated colon cancer. Cancer Res. 74, 6150-6160. doi: 10.1158/0008-5472.CAN-14-0870

Feijoo, C., Campbell, D. G., Jakes, R., Goedert, M., and Cuenda, A. (2005). Evidence that phosphorylation of the microtubule-associated protein Tau by SAPK4/p38delta at Thr50 promotes microtubule assembly. J. Cell Sci. 118, 397-408. doi: 10.1242/jcs.01655

Gaestel, M., Kotlyarov, A., and Kracht, M. (2009). Targeting innate immunity protein kinase signalling in inflammation. Nat. Rev. Drug Discov. 8, 480-499. doi: $10.1038 / \mathrm{nrd} 2829$

Gantke, T., Sriskantharajah, S., and Ley, S. C. (2011). Regulation and function of TPL-2, an I $\kappa$ B kinase-regulated MAP kinase kinase kinase. Cell Res. 21, 131-145. doi: 10.1038/cr.2010.173

Gillespie, M. A., Le Grand, F., Scimè, A., Kuang, S., von Maltzahn, J., Seale, V., et al. (2009). p38-\{gamma\}-dependent gene silencing restricts entry into the myogenic differentiation program. J. Cell Biol. 187, 991-1005. doi: 10.1083/jcb.200907037

Goedert, M., Cuenda, A., Craxton, M., Jakes, R., and Cohen, P. (1997). Activation of the novel stress-activated protein kinase SAPK4 by cytokines and cellular stresses is mediated by SKK3 (MKK6); comparison of its substrate specificity with that of other SAP kinases. EMBO J. 16, 3563-3571. doi: 10.1093/emboj/16.12.3563

González-Terán, B., Cortés, J. R., Manieri, E., Matesanz, N., Verdugo, A., Rodríguez, M. E., et al. (2013). Eukaryotic elongation factor 2 controls TNFalpha translation in LPS-induced hepatitis. J. Clin. Invest. 123, 164-178. doi: 10.1172/JCI65124

González-Terán, B., López, J. A., Rodríguez, E., Leiva, L., Martínez-Martínez, S., Bernal, J. A., et al. (2016). p38gamma and delta promote heart hypertrophy by targeting the mTOR-inhibitory protein DEPTOR for degradation. Nat. Commun. 7:10477. doi: 10.1038/ncomms 10477

Hanahan, D., and Weinberg, R. A. (2011). Hallmarks of cancer: the next generation. Cell 144, 646-674. doi: 10.1016/j.cell.2011.0 2.013 
Hasegawa, M., Cuenda, A., Spillantini, M. G., Thomas, G. M., Buee-Scherrer, V., Cohen, P., et al. (1999). Stress-activated protein kinase-3 interacts with the PDZ domain of alpha1-syntrophin. A mechanism for specific substrate recognition. J. Biol. Chem. 274, 12626-12631. doi: 10.1074/jbc.274.18.12626

Hou, S. W., Zhi, H. Y., Pohl, N., Loesch, M., Qi, X. M., Li, R. S., et al. (2010). PTPH1 dephosphorylates and cooperates with p38gamma MAPK to increase ras oncogenesis through PDZ-mediated interaction. Cancer Res. 70, 2901-2910. doi: 10.1158/0008-5472.CAN-09-3229

Ittner, A., Block, H., Reichel, C. A., Varjosalo, M., Gehart, H., Sumara, G., et al. (2012). Regulation of PTEN activity by p38delta-PKD1 signaling in neutrophils confers inflammatory responses in the lung. J. Exp. Med. 209, 2229-2246. doi: 10.1084/jem.20120677

Iwasaki, A., and Medzhitov, R. (2010). Regulation of adaptive immunity by the innate immune system. Science 327, 291-295. doi: 10.1126/science.1183021

Jiang, Y., Gram, H., Zhao, M., New, L., Gu, J., Feng, L., et al. (1997). Characterization of the structure and function of the fourth member of p38 group mitogen-activated protein kinases, p38delta. J. Biol. Chem. 272, 30122-30128. doi: 10.1074/jbc.272.48.30122

Kawai, T., and Akira, S. (2007). TLR signaling. Semin. Immunol. 19, 24-32. doi: 10.1016/j.smim.2006.12.004

Knebel, A., Morrice, N., and Cohen, P. (2001). A novel method to identify protein kinase substrates: eEF2 kinase is phosphorylated and inhibited by SAPK4/p38delta. EMBO J. 20, 4360-4369. doi: 10.1093/emboj/20.16.4360

Kuma, Y., Sabio, G., Bain, J., Shpiro, N., Márquez, R., and Cuenda, A. (2005). BIRB796 inhibits all p38 MAPK isoforms in vitro and in vivo. J. Biol. Chem. 280, 19472-19479. doi: 10.1074/jbc.M414221200

Linares, J. F., Duran, A., Reina-Campos, M., Aza-Blanc, P., Campos, A., Moscat, J., et al. (2015). Amino acid activation of mTORC1 by a PB1domain-driven kinase complex cascade. Cell Rep. 12, 1339-1352. doi: 10.1016/j.celrep.2015.07.045

Mertens, S., Craxton, M., and Goedert, M. (1996). SAP kinase-3, a new member of the family of mammalian stress-activated protein kinases. FEBS Lett. 383, 273-276. doi: 10.1016/0014-5793(96)00255-4

Perdiguero, E., Ruiz-Bonilla, V., Gresh, L., Hui, L., Ballestar, E., Sousa-Victor, P., et al. (2007). Genetic analysis of p38 MAP kinases in myogenesis: fundamental role of p38alpha in abrogating myoblast proliferation. EMBO J. 26, 1245-1256. doi: 10.1038/sj.emboj.7601587

Pogozelski, A. R., Geng, T., Li, P., Yin, X., Lira, V. A., Zhang, M., et al. (2009). p38gamma mitogen-activated protein kinase is a key regulator in skeletal muscle metabolic adaptation in mice. PLoS ONE 4:e7934. doi: 10.1371/journal.pone.0007934

Ramachandra, C. J., Mehta, A., Wong, P., and Shim, W. (2016). ErbB4 Activated p38gamma MAPK isoform mediates early cardiogenesis through NKx2.5 in human pluripotent stem cells. Stem Cells 34, 288-298. doi: 10.1002/stem.2223

Remy, G., Risco, A. M., Iñesta-Vaquera, F. A., González-Terán, B., Sabio, G., Davis, R. J., et al. (2010). Differential activation of p38MAPK isoforms by MKK6 and MKK3. Cell. Signal. 22, 660-667. doi: 10.1016/j.cellsig.2009.11.020
Risco, A., and Cuenda, A. (2012). New insights into the p38gamma and p38delta MAPK pathways. J. Signal Transduct. 2012:520289. doi: 10.1155/2012/5 20289

Risco, A., del Fresno, C., Mambol, A., Alsina-Beauchamp, D., MacKenzie, K. F., Yang, H. T., et al. (2012). p38gamma and p38delta kinases regulate the Toll-like receptor 4 (TLR4)-induced cytokine production by controlling ERK1/2 protein kinase pathway activation. Proc. Natl. Acad. Sci. U.S.A. 109, 11200-11205. doi: 10.1073/pnas.1207290109

Sabio, G., Arthur, J. S., Kuma, Y., Peggie, M., Carr, J., Murray-Tait, V., et al. (2005). p38gamma regulates the localisation of SAP97 in the cytoskeleton by modulating its interaction with GKAP. EMBO J. 24, 1134-1145. doi: 10.1038/sj.emboj.7600578

Sabio, G., Cerezo-Guisado, M. I., Del Reino, P., Iñesta-Vaquera, F. A., Rousseau, S., Arthur, J. S., et al. (2010). p38gamma regulates interaction of nuclear PSF and RNA with the tumour-suppressor hDlg in response to osmotic shock. J. Cell Sci. 123, 2596-2604. doi: 10.1242/jcs.066514

Sabio, G., Reuver, S., Feijoo, C., Hasegawa, M., Thomas, G. M., Centeno, F., et al. (2004). Stress- and mitogen-induced phosphorylation of the synapse-associated protein SAP90/PSD-95 by activation of SAPK3/p38gamma and ERK1/ERK2. Biochem. J. 380, 19-30. doi: 10.1042/bj20031628

Schindler, E. M., Hindes, A., Gribben, E. L., Burns, C. J., Yin, Y., Lin, M. H., et al. (2009). p38delta Mitogen-activated protein kinase is essential for skin tumor development in mice. Cancer Res. 69, 4648-4655. doi: 10.1158/00085472.CAN-08-4455

Sumara, G., Formentini, I., Collins, S., Sumara, I., Windak, R., Bodenmiller, B., et al. (2009). Regulation of PKD by the MAPK p38delta in insulin secretion and glucose homeostasis. Cell 136, 235-248. doi: 10.1016/j.cell.2008.1 1.018

Tortorella, L. L., Lin, C. B., and Pilch, P. F. (2003). ERK6 is expressed in a developmentally regulated manner in rodent skeletal muscle. Biochem. Biophys. Res. Commun. 306, 163-168. doi: 10.1016/S0006-291X(03)00936-7

Zur, R., Garcia-Ibanez, L., Nunez-Buiza, A., Aparicio, N., Liappas, G., Escós, A., et al. (2015). Combined deletion of p38gamma and p38delta reduces skin inflammation and protects from carcinogenesis. Oncotarget 6, 12920-12935. doi: 10.18632 /oncotarget. 4320

Conflict of Interest Statement: The authors declare that the research was conducted in the absence of any commercial or financial relationships that could be construed as a potential conflict of interest.

Copyright (C) 2016 Escós, Risco, Alsina-Beauchamp and Cuenda. This is an openaccess article distributed under the terms of the Creative Commons Attribution License (CC BY). The use, distribution or reproduction in other forums is permitted, provided the original author(s) or licensor are credited and that the original publication in this journal is cited, in accordance with accepted academic practice. No use, distribution or reproduction is permitted which does not comply with these terms. 